\title{
Secondary neoplasm of the thyroid presenting as a goitre
}

\author{
J. N. HAR COURT-WEBSTER \\ From the Department of Pathology, University of Edinburgh
}

SYNOPSIS Secondary neoplasm is a very rare finding in a goitre despite the numerous reports metastatic deposits in the thyroid gland at necropsy. Eleven cases are reported of such deposit; presenting clinically as a thyroid nodule, the sites of origin including a meningioma and an unusuat carcinoma of the skin. Some of the factors, including pre-existent degenerative processes, believedo to determine the incidence of secondary neoplasm in the thyroid, are discussed together with the problems of establishing the diagnosis.

The thyroid gland has long been recognized, despite its great vascularity, as one of the less common sites for secondary neoplasm. There is, however, some variation in the incidence at necropsy. Abrams, Spiro, and Goldstein (1950) recorded $19(0 \cdot 19 \%)$ thyroid metastatic deposits in 1,000 consecutive cases of disseminated carcinoma whilst Willis (1931) described nine $(5 \cdot 2 \%)$ among 170 similar cases, and Shimoaka, Sokal, and Pickren (1962) reported another $188(8.6 \%)$ such deposits in 1,980 examples of malignant disease. The deposits are derived from a wide variety of neoplasms, the lungs, melanomata, the breasts, and the kidneys being the most frequently recorded sites of origin.

In contrast to the numerous records of metastatic deposits in the thyroid at necropsy they are only rarely reported presenting clinically as a swelling. The true diagnosis may be suspected from the history but frequently the deposit simulates a primary carcinoma; occasionally they are an unexpected finding in an involutionary or degenerative goitre.

This paper records 11 cases of goitre in which there were secondary deposits, five mimicking a primary carcinoma, from a variety of different sources.

\section{CASE REPORTS}

CASE 1 A 56-year-old woman presented with a one-year history of a slowly enlarging, firm, slightly mobile nodule in the right lobe of the thyroid. It was regarded as a degeneration cyst and excised.

Some years previously the right breast had been removed for a grade I carcinoma; subsequently the patient remained well.

Received for publication 6 August 1964.
Histology The nodule was a poorly differentiated, polygonal cell adenocarcinoma of similar structure to theff reported in the breast. The carcinoma was almost en closed by a capsule of connective tissue and clearly distinguished from the adjacent normal thyroid tissue There was no evidence indicating the route of spread to the thyroid.

The subsequent history of the patient is unknown.

CASE 2 A 58-year-old man, who one year previously haf undergone a right pneumonectomy for carcinoma gradually developed a firm swelling of the right lobe of the thyroid together with increasing dyspnoea and dysphagia. A metastatic deposit was suspected and partiai thyroidectomy performed to relieve the symptoms. Theze was no lymphadenopathy.

Histology The right lobe of the thyroid was extensive infiltrated by a moderately well-differentiated squamos cell carcinoma similar to that in the right lung. There was no evidence of pre-existent degeneration changes in the thyroid.

The patient died shortly after the thyroidectomy be there was no necropsy.

CASE 3 A 39-year-old woman, with a six-month histo of progressively increasing dysphagia and dyspnoea, w⿳亠口冋丸 found to have a predominantly left-sided nodular collow goitre. There was no evidence of disease in the other systems. Partial thyroidectomy was performed.

Histology There was extensive replacement of the enlarged left lobe by a sharply defined, clear cell adenocarcinoma; there was no evidence of a transition between the adjacent normal thyroid vesicles and the carcinong and there was a minimal stromal reaction.

The tentative diagnosis of a metastatic deposit from renal carcinoma was confirmed at necropsy six mont bs later; death was attributed to bronchopneumonia. Therfe were no other secondary deposits. 
CASE 4 A 40-year-old woman presented with a onemonth history of a limp and pain in the left hip. On examination the principal finding was a moderately enlarged, firm thyroid with displacement of the trachea to the right. Radiography demonstrated an osteolytic lesion centred in the left ilium and roof of the acetabulum but there was no abnormality of the spine, long bones, or lungs. A diagnosis of carcinoma of the thyroid with a bone metastasis was made and a biopsy taken from the thyroid.

Histology Both lobes included several, variably sized microfollicular adenomata. Partially replacing some of the adenomata and the neighbouring tissue was a pleomorphic, clear cell, solid alveolar carcinoma (Fig. 1); there was an abrupt transition between it and the thyroid parenchyma but no significant stromal reaction.

The provisional histological diagnosis of a metastatic deposit from a renal carcinoma was confirmed after a retrograde pyelogram had demonstrated a still symptomless mass in the right kidney. Death occurred within eight months, there being multiple metastatic deposits in the bones and lungs.

CASE 5 A 66-year-old woman was first seen with a one-month history of a small, ulcerated nodule on the

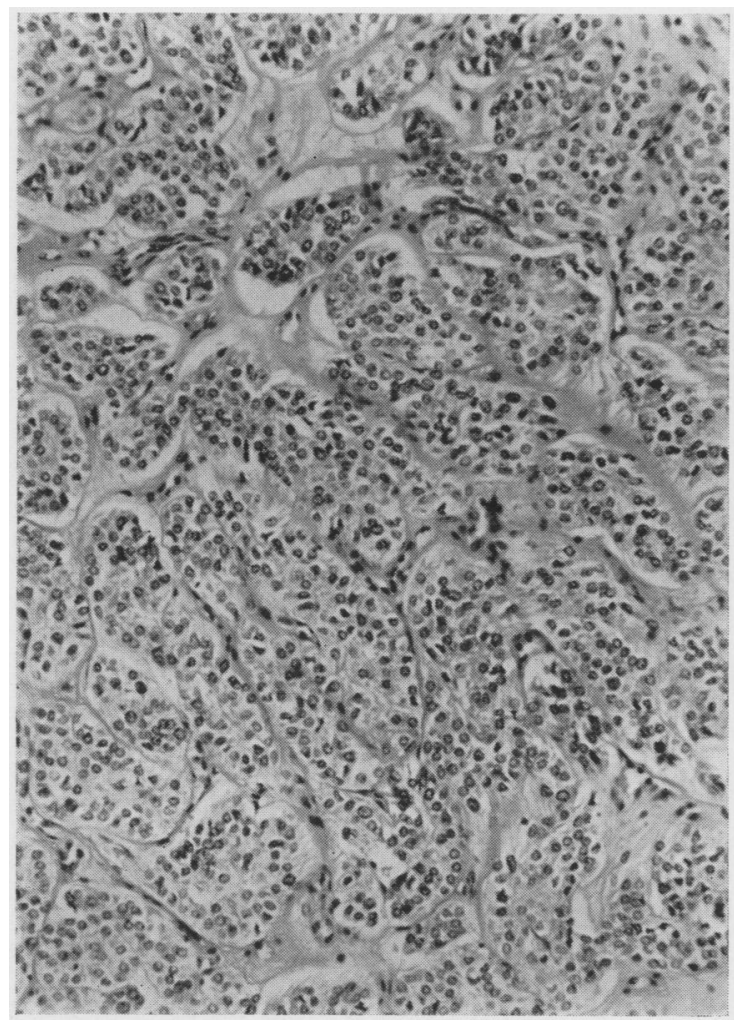

FIG. 1. Case 4: A large metastasis from a renal carcinoma which presented as a goitre. Haematoxylin and eosin $\times$ 140. lateral aspect of the right thigh. Four months later a firm, nodular swelling appeared in the left lobe of the thyroid together with supraclavicular and inguinal lymphadenopathy; the skin nodule remained unchanged. The provisional diagnosis was carcinoma of the thyroid with metastatic deposits.

Histology Biopsies of the thyroid swelling, the supraclavicular lymph nodes, and the nodule on the thigh all showed a keratin-forming, squamous cell carcinoma. However, that from the skin and, to a lesser degree, that from the thyroid, also included large infiltrating foci of pleomorphic and actively mitotic spindle cells arranged, for the most part, in parallel rows (Fig. 2). Many of these cells contained small quantities of brown pigment some of which was demonstrated to be melanin (Fontana's method) and some to be haemosiderin (Perls's Prussian blue method). In several areas and particularly at the edges of the skin nodule, where an origin from the epidermis could be traced, there was a gradual transition between the squamous and the pigmented spindle cells (Fig. 3).There was no evidence of involutionary changes in the thyroid.

The melano-squamous carcinoma of the skin was regarded as the primary neoplasm with metastatic deposits in the thyroid and lymph nodes.

Over the ensuing 10 weeks there was increasing cervical lymphadenopathy followed by a respiratory infection from which she died: no necropsy was obtained.

CASE 6 A 52-year-old man presented with a firm, immobile swelling in the right lobe of the thyroid; there was no evidence of cervical lymphadenopathy. It was regarded as a carcinoma and a complete excision of the right lobe was attempted.

Nine years previously a tumour had been removed from the falx cerebri of the parietal area. This had recurred after seven years and removal was again attempted. On both occasions the histological interpretation had been difficult but the tumour was considered to be an active, vascular meningioma. ${ }^{1}$

Histology There was extensive infiltration of the right lobe of the thyroid by sheets of undifferentiated, pleomorphic polygonal cells (Fig. 4), amongst which were numerous vascular channels. The neoplastic cells abutting upon the endothelial lining of the sinusoids were frequently fusiform but there were few giant cells. There were numerous bizarre and hyperchromatic nuclei and moderate numbers of mitotic figures (Fig. 5). Only scattered, small foci of thyroid tissue remained and there was no stromal reaction.

A diagnosis of an undifferentiated malignant neoplasm of undetermined histogenesis was made; at this stage the detailed previous history and histological sections of the meningioma were not available.

Necropsy, eight months later, showed multiple small tumour deposits in the meninges close to the site of excision of the meningioma and in the lungs but there were none elsewhere. The histological features of both these deposits and the thyroid tumour closely resembled

${ }^{1}$ A detailed account of the histological findings will be published by Drs. A. Gordon and A. F. J. Moloney. J. Neurol (in press). 


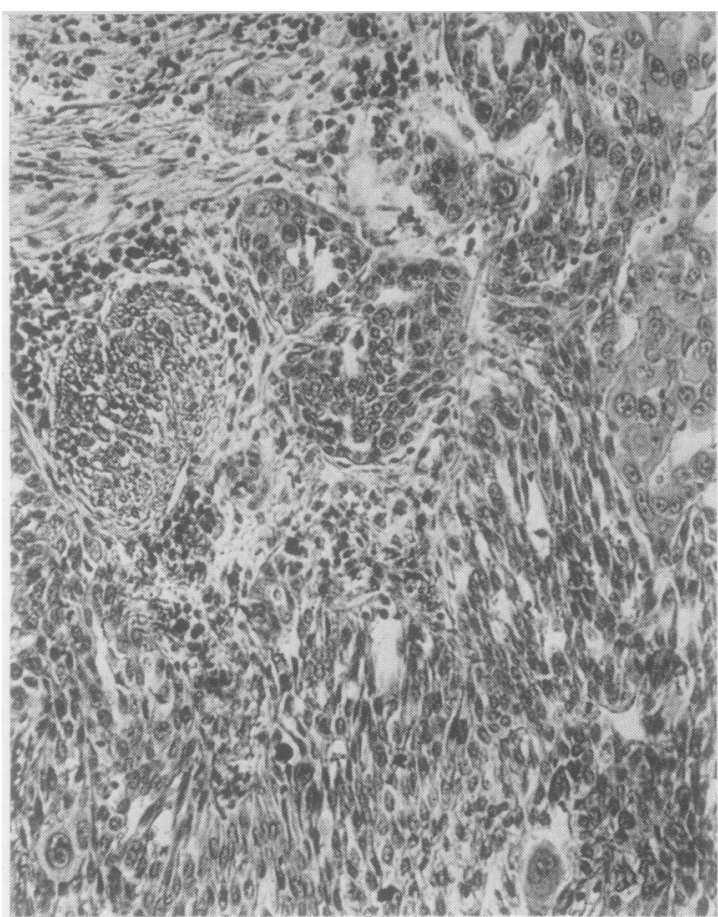

FIG. 2. Case 5: Part of the skin nodule showing large numbers of spindle cells (pigment not shown) arranged in parallel rows amongst which are scattered clusters of pleomorphic squamous cells. Haematoxylin and eosin $\times$ 140.

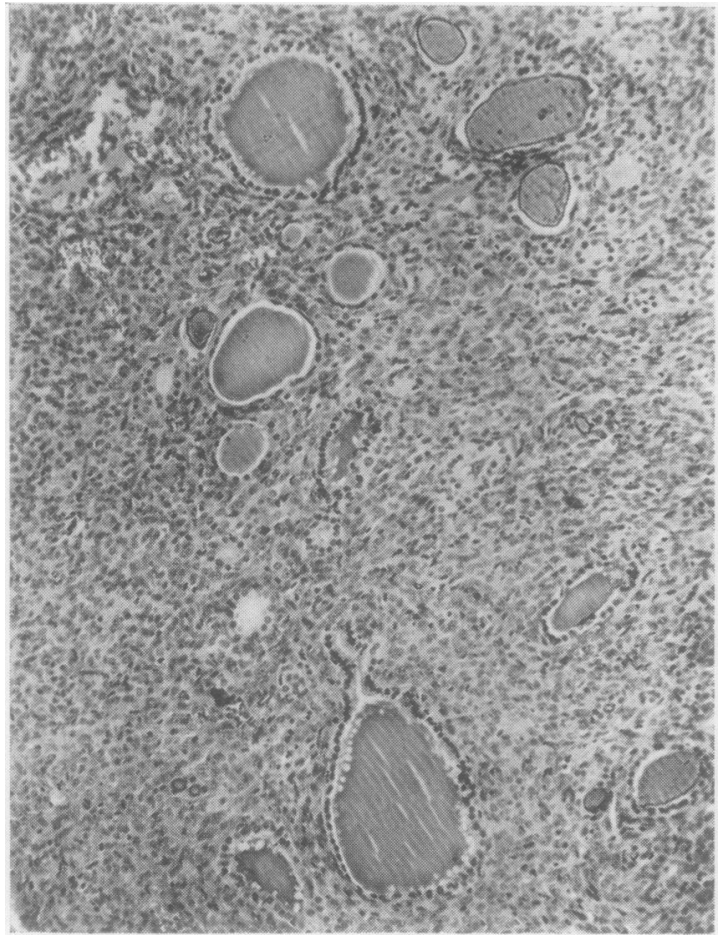

FIG. 4

FIG. 4 .

FIG. 4. Case 6: A deposit from an undifferentiated meningioma of the falx replacing much of the right lobe
the thyroid. Haematoxylin and eosin $\times 100$. FIG. 5. Case 6: Higher-power view of Figure 4 to show the pleomorphic polygonal cell structure of the deposif Note the fusiform character of many of the cells adjacent to the endothelium of the vascular channels. There are numerous bizarre and hyperchromatic nuclei in addition to mitotic figures. Haematoxylin and eosin $\times 300$.

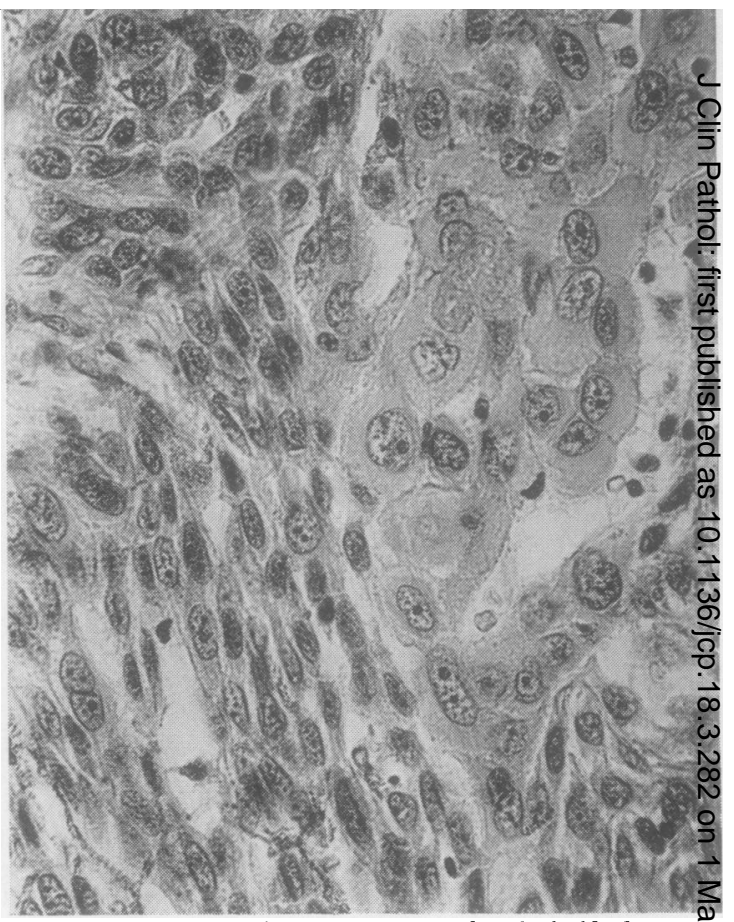

FIG. 3. Case 5: Higher-power view of right half of Figure 2. Note the transition between, right, the pleomorphib and bizarre squamous cells and, left, the pleomorph舁 spindle cells in which fine granules of pigment can be seen Haematoxylin and eosin $\times 350$.

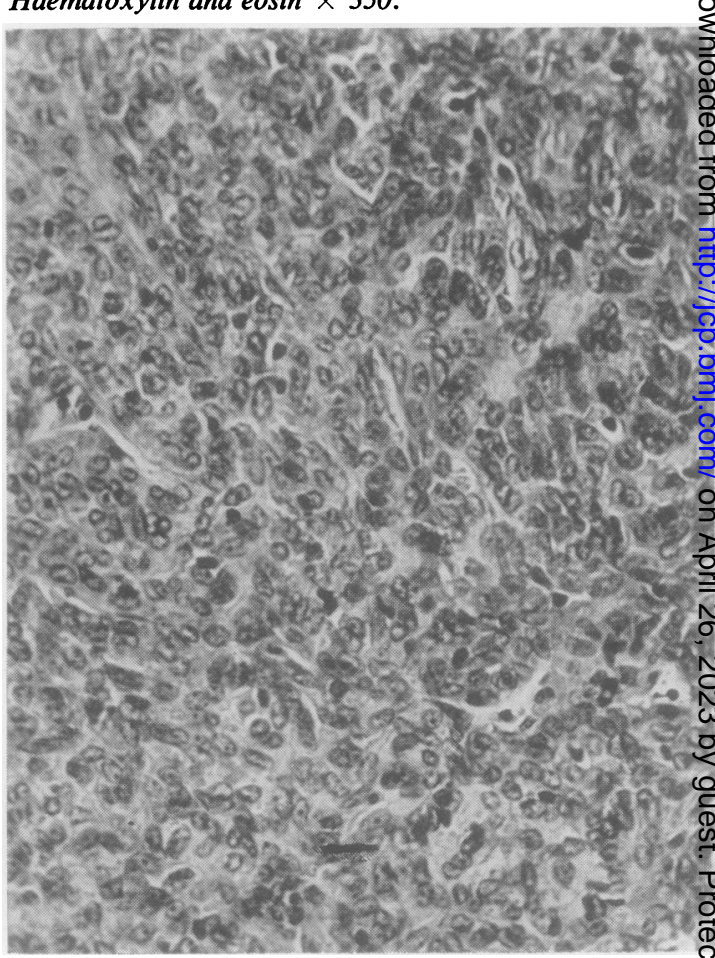

FIG. 5 


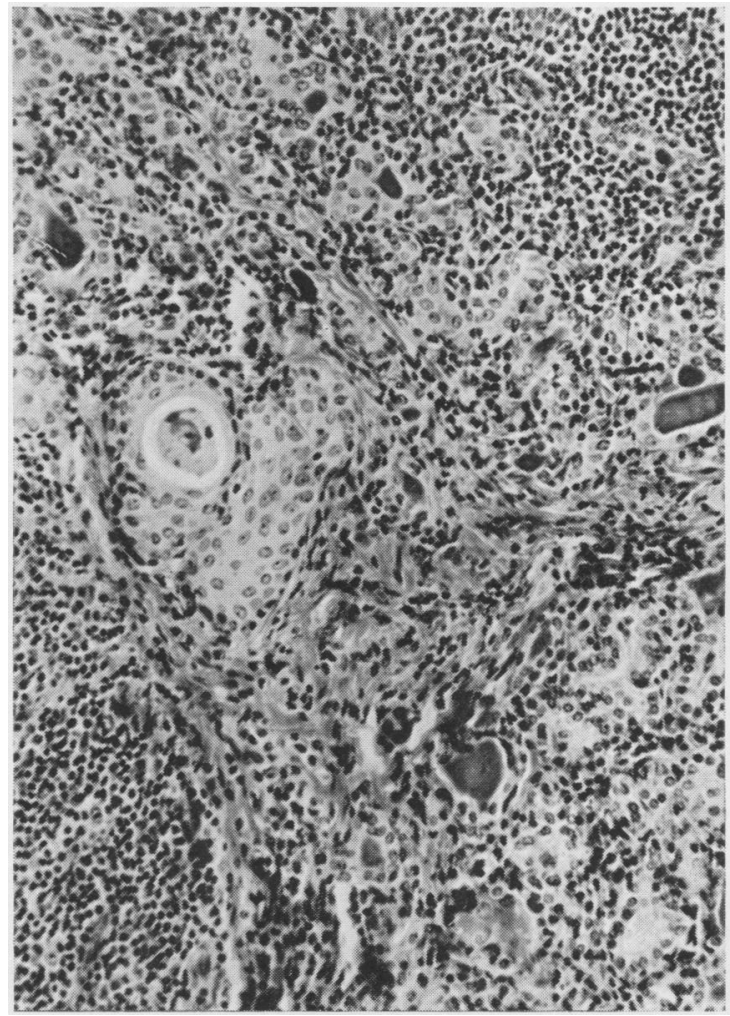

FIG. 6. Case 7: A solitary metastatic deposit from an epidermoid carcinoma of the cervix uteri in a thyroid also showing the features of Hashimoto's disease. Haematoxylin and eosin $\times 300$.

those of the previously removed meningioma which was concluded to be the site of origin.

CASE 7 A 52-year-old married woman presented with a history of vaginal bleeding and a firm, symmetrical swelling in the neck associated with discomfort and dysphagia. After a provisional diagnosis of carcinoma of the cervix uteri with a metastatic deposit in the thyroid gland, both biopsy of the cervix and a partial thyroidectomy were performed.

Histology The cervical specimen was a moderately well-differentiated epidermoid carcinoma. The thyroid showed the features of Hashimoto's disease; however, a microscopic deposit of carcinoma similar to that in the cervix was seen in the isthmus (Fig. 6). Metastatic carcinoma also infiltrated a supraclavicular lymph node removed at thyroidectomy.

CASE 8 An otherwise fit 21-year-old man was seen with a small, cutaneous nodule on the right buttock thought to have been present since birth but recently enlarging. There was also a small, mobile lump in the left lobe of the thyroid believed to have gradually appeared over the previous nine months. Both were excised.
Histology The nodule from the buttock showed the features of the moderately pigmented malignant melanoma. The thyroid nodule was a macro-follicular adenoma; a small focus of melanin-pigmented (Fontana's method) pleomorphic spindle cells, identical with those seen in the melanoma of the buttock, was however found amongst the colloid-laden follicles of the adenoma. It was regarded as a secondary deposit.

The patient remained well with no evidence of any further metastatic deposits one year later.

CASE 9 A 74-year-old woman had a slightly pigmented malignant melanoma excised from the left forearm. Three years later the right lobe of the thyroid became moderately enlarged but there was no evidence, at the site of excision, of any recurrence. A biopsy of the thyroid was taken.

Histology The thyroid parenchyma was infiltrated by non-pigmented fusiform and polyhedral cells, including small giant forms, arranged in interlacing bundles with a scanty but vascular fibrous stroma. Despite the absence of pigment the similar cytological features of the previously excised melanoma and the thyroid biopsy indicated that the latter was a metastatic deposit.

Over the next few months numerous enlarged lymph nodes appeared in the neck and axillae, death following from a respiratory infection. No necropsy was performed.

CASE 10 A 27-year-old woman presented with discomfort and dysphagia attributed to a nodular goitre. There were no symptoms or signs of abnormality in the other systems. Partial thyroidectomy was performed for the relief of the symptoms.

Histology The thyroid was extensively infiltrated by sheets of undifferentiated, small, dark-staining round cells similar to but slightly larger than lymphocytes. There was no stromal reaction nor was there any other abnormality of the thyroid. The tumour was provisionally regarded as a lymphosarcoma.

Necropsy, a few weeks later, showed an adrenal swelling together with a moderate sized mediastinal mass and a deposit in the cavity of the right auricular appendage of the heart. These all showed similar histological features to the thyroid tumour but also included small numbers of rosette formations; a diagnosis of an adrenal neuroblastoma with metastatic deposits was made.

CASE 11 An otherwise healthy 50-year-old woman was seen with a nodular colloid goitre which was treated by partial thyroidectomy.

Histology In many areas the thyroid showed the classical features of a nodular goitre; however, one area included a small focus of a poorly differentiated, cuboidal cell adenocarcinoma demarcated from the adjacent thyroid tissue by a thin capsule of connective tissue (Fig. 7). The structure of the tumour and the lack of any transition between it and the thyroid vesicles suggested secondary neoplasm.

A small nodule was eventually found in one of the breasts and excised; this proved to be a typical adenocarcinoma of the breast and was consequently regarded as the primary. 


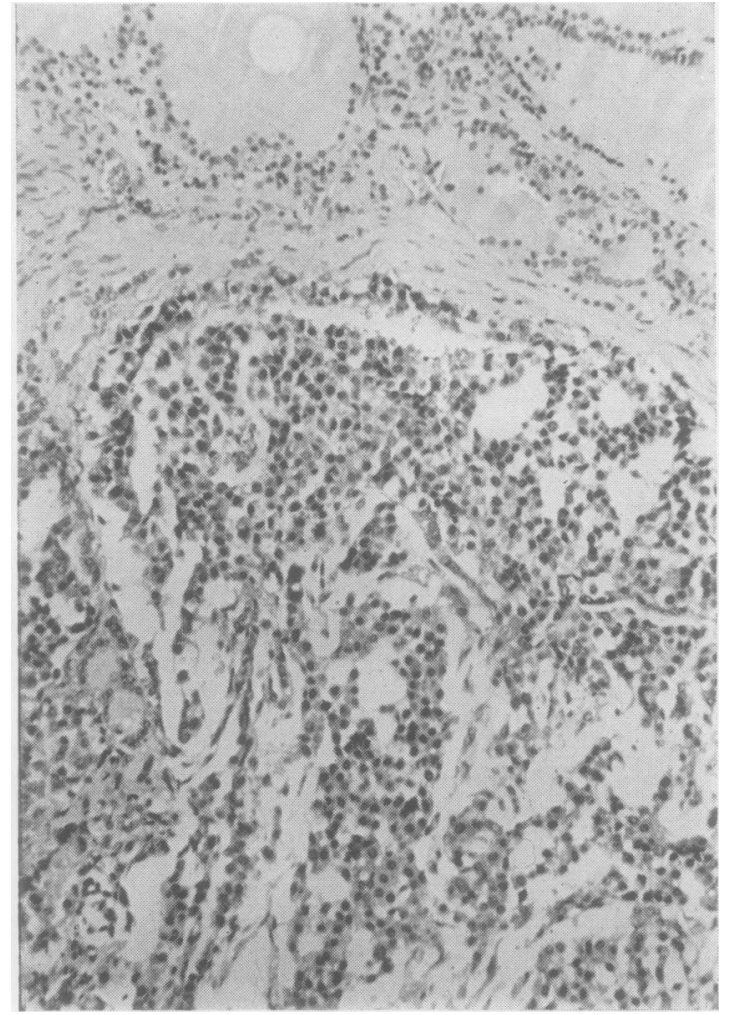

FIG. 7. Case 11: A deposit of poorly differentiated adenocarcinoma from the breast unexpectedly found in a nodular colloid goitre. Haematoxylin and eosin $\times 120$.

Necropsy six months later demonstrated extensive infiltration of the liver and the bones by deposits of a similar carcinoma.

\section{DISCUSSION}

Elliott and Frantz (1960) reported 14 cases of goitre caused by secondary neoplasm and reviewed a further 44 found in the literature. Four more examples have since been recorded (Heimann, 1961; Roberts, 1962). The infrequency of goitre attributable to this cause is surprising even when compared with the comparatively low incidence of metastatic deposits in the thyroid at necropsy. This discrepancy may, at least in part, be attributed to the high proportion (over $50 \%$ ) of microscopic deposits in many necropsy series: 'only careful microscopy will detect all deposits' (Willis, 1931). A factor determining this apparent natural resistance of the thyroid is probably its high oxygen tension and iodine content which retards the establishment and multiplication of malignant cells with their relatively anaerobic metabolism. The frequent association of the metastatic deposits, both in this and other series (Willis, 1952;
Bruce and Michie, 1954), with atrophic and de्ञ generative conditions of the thyroid, in which the oxygen tension and iodine content are probab $\overrightarrow{\mathrm{F}_{\mathrm{y}}}$ reduced, supports this belief.

The neoplasms recorded, in clinical cases, metastasizing to the thyroid are shown in the Table

TABLE

METASTATIC NEOPLASM FOUND IN GOITRE

\begin{tabular}{lccr} 
Primary Site & $\begin{array}{c}\text { No. of Cases } \\
\text { in Literature }\end{array}$ & $\begin{array}{c}\text { Author's } \\
\text { Cases }\end{array}$ & Total \\
\hline Kidney & 35 & 2 & 37 \\
Alimentary tract & 9 & 0 & 9 \\
Lung & 8 & 1 & 9 \\
Breast & 6 & 2 & 8 \\
Skin \{ $\left\{\begin{array}{l}\text { squamous } \\
\text { malignant melanoma }\end{array}\right.$ & 1 & 0 & 1 \\
Adrenal & 1 & 2 & 3 \\
Adrenal neuroblastoma & 0 & 1 & 1 \\
Liver & 1 & 0 & 1 \\
Meningioma & 0 & 1 & 1 \\
Cervix uteri & 1 & 0 & 1 \\
Total & 0 & 1 & 1 \\
\end{tabular}

${ }^{1}$ Carcinoma unless otherwise stated

The preponderance of renal cancer contrasts with the finding in most necropsy series in which the lungs, melanomata, the breasts, and the kidneys as almost equal in their frequency as the site of origio. This is of interest when considering the route of spread to the thyroid, for Willis (1952) considess entry via the lymphatics to be very rare in this orga庐; the predilection of renal cancer for haematogenows distribution is well known. The more equal distrib tion of origin in necropsy reports may be explained by the dissemination of malignant cells via the bloox stream as a near terminal event in many cancers. :-

The skin, with the exception of melanomata, is $\overline{3}$ very rare source of secondary neoplasm in the thyroid. The carcinoma with mixed squamous am melanotic features (case 5) is therefore of interest in that whilst its histogenesis remains in doubt, although it is tempting to regard it as an example of what has been termed 'melanogenic metaplasin' (Shivas and MacLennan, 1963), its spread to the thyroid is more in keeping with the behaviour of $\mathrm{n}$ a melanoma than that of an epithelioma. The menio gioma (case 6) is also of unusual interest; these nefplasms are occasionally recorded as metastasizing to various tissues, including the lungs (Jurow, 1941) a ad the liver (Hamblet, 1944), but there is no previoßs report of spread to the thyroid.

Secondary neoplasm in the thyroid is an indicatgr of grave prognosis; in seven of the nine patienifs being reported, whose subsequent history is know metastatic deposits were identified at other sifes either before or a few months after biopsy of the thyroid. This is in accord with the observation that 
spread to the thyroid at necropsy is virtually unknown in the absence of multiple deposits elsewhere (Bruce and Michie, 1954; Mortensen, Woolner, and Bennett, 1956).

The recognition of a metastatic deposit in the thyroid, when in association with a previously identified neoplasm, may present no particular difficulty to the histopathologist. In the absence of such a history or with a poorly differentiated deposit there may, however, be considerable difficulty but nevertheless, as in this and previous reports (H'Doubler, 1936; Elliott and Frantz, 1960), the possibility of a secondary deposit mimicking a primary carcinoma of the thyroid should not be overlooked. Particular care must be exercised in the diagnosis of a deposit from a renal carcinoma because of the histological similarity between the latter and the primary clear cell carcinoma occasionally seen in the thyroid (Chesky, Hellwig, and Barbosa, 1957). On the other hand, squamous cell carcinoma arising in the thyroid is very rare (I have seen one amongst 359 carcinomas of this organ) and the recognition of this type of neoplasm should arouse the suspicion of it being a metastatic deposit. In the final analysis the differentiation between a primary and a second- ary neoplasm will often be made only by careful clinico-pathological consultation.

I wish to thank Professor G. L. Montgomery for criticism and advice and Mr. T. C. Dodds, Director of the Department of Medical Photography, for the illustrations. Grateful thanks are also due to Professor R. McWhirter, Mr. J. R. Cameron and Dr. J. M. Drennan for assistance in collection of the cases.

\section{REFERENCES}

Abrams, H. L., Spiro, R., and Goldstein, N. (1950). Cancer (Philad.), 3,74

Bruce, G. G., and Michie, W. (1954). Brit. J. Surg., 42, 257.

Chesky, V. E., Hellwig, C. A., and Barbosa, E. (1957). Surgery, 42, 282.

Elliott, R. H. E. Jr., and Frantz, V. K. (1960). Ann. Surg., 151, 551. Hamblet, J. B. (1944). Arch. Path., 37, 216.

H'Doubler, F. T. (1936). In Medical Papers Dedicated to H. A. Christian, p. 791. Waverly Press, Baltimore.

Heimann, P. (1961). Nord. méd., 65, 752.

Jurow, H. N. (1941). Arch. Path., 32, 222.

Mortensen, J. D., Woolner, L. B., and Bennett, W. A. (1956). Cancer (Philad.), 9, 306.

Roberts, L. (1962). Postgrad. med. J., 38, 162.

Shimoaka, K., Sokal, J. E., and Pickren, J. W. (1962). Cancer (Philad.), $15,557$.

Shivas, A. A., and MacLennan, W. D. (1963). Brit. J. Cancer, 17, 411. Willis, R. A. (1931). Amer. J. Path., 7, 187.

- (1952). The Spread of Tumours in the Human Body, 2nd ed., p. 271. Butterworth, London. 